\title{
Morphologies and Substructures of UV Star-Forming Galaxies at Intermediate-z
}

\author{
E. N. Voyer ${ }^{1,2}$, D. F. de Mello ${ }^{2,3}$, S. M. Blevins ${ }^{2}$, H. I. Teplitz ${ }^{4}$, \\ J. P. Gardner ${ }^{3}$, B. D. Siana ${ }^{5}$, and E. Soto ${ }^{2}$ \\ ${ }^{1}$ Aix Marseille Université, CNRS, LAM, UMR 7326, 13388 Marseille, France \\ ${ }^{2}$ The Catholic University of America, Physics Department, Washington, DC 20064, USA \\ ${ }^{3}$ Observational Cosmology Laboratory, Code 665, NASA's GSFC, Greenbelt, MD 20771, USA \\ ${ }^{4}$ Infrared Processing and Analysis Center, Caltech, Pasadena, CA 91125, USA \\ ${ }^{5}$ Department of Physics and Astronomy, University of California, Riverside, CA 92521, USA
}

\begin{abstract}
Exploring potential links between the internal physical processes of galaxies with respect to their external morphologies can reveal connections between past and present populations. One primary physical driver of galaxy evolution is star formation, which is directly detected from UV emission. Here, we summarize a study investigating the optical and UV morphologies of rest-frame UV-detected star-forming galaxies at intermediate redshifts $(0.1<\mathrm{z}<1.2)$ observed with the Hubble Space Telescope (HST) Solar Blind Channel (far-UV) and Wide Field PlanetaryCamera 2 (WFPC2; U-band) in the Great Observatories Origins Deep Survey fields.
\end{abstract}

Keywords. ultraviolet: galaxies — galaxies: structure — galaxies: evolution

- Method. Our sample consists of 230 galaxies detected in the FUV and 96 in the Uband (Teplitz 2006; Voyer 2009, 2011). We examine the rest-frame optical morphologies of the 230 FUV sources by measuring their Sérsic indices with GALFIT, comparing them to their SED based galaxy spectral types (Dahlen et al. 2010), and to the $\mathrm{z}=0$ star-forming galaxy population. Secondly, we measure the physical sizes of rest-UV sub-galactic starforming clumps in a sub-set of 18 HUDF galaxies with clumpy UV morphologies and compare them to the $\mathrm{z}=2-3$ rest-UV clumpy population (Elmegreen \& Elmegreen 2005).

- Results. We find that unobscured star formation is primarily occuring in exponential disk and merger-like optical morphologies over intermediate-z. The rest-UV star-forming clumps range between $1.9-8.5 \mathrm{kpc}$ in physical size, which is $\sim 2-4$ times larger than clumps measured at $\mathrm{z}>2(1-2 \mathrm{kpc})$. We suggest the clumpy rest-UV nature of star-formation at intermediate-z may be a signature of mechanisms driving transitions in rest-frame optical morphologies over this epoch from clumpy tidal/merger-like to exponential disks.

- Ongoing Work. Recently, the HST Wide Field Camera 3 (WFC3) UV survey of the HUDF was completed (P.I. Teplitz). A preliminary study of these data reveals that, in a few cases, a singly measured WFPC2 clump is resolved into multiple WFC3 clumps due to its superior resoultion. Tests on the WFPC2 image resolution predict that the new WFC3 data will reveal more 1-2 kpc clumps, resulting in a smaller increase in average UV clump size from intermediate- to high-z $(\sim 1.6-3$ times larger $)$ than we currently measure.

\section{References}

Dahlen, T., Mobasher, B., Dickinson, M., et al. 2010 ApJ, 724, 425

Elmegreen, B. G. \& Elmegreen, D. M. 2005, ApJ, 627, 632

Teplitz, H. I., Siana, B., Brown, T. M., et al. 2006, AJ, 132, 853

Voyer, E. N., de Mello, D. F., Siana, B., et al. 2009, AJ, 138, 598

Voyer, E. N., Gardner, J. P., Teplitz, H. I., Siana, B. D., \& de Mello, D. F. 2011, ApJ, 736, 80 